\title{
Fundamentals of a continuum theory of dislocations
}

\author{
Thomas Hochrainer*† \\ Universität Karlsruhe (TH), Institut für Zuverlässigkeit von Bauteilen und Systemen, \\ Kaiserstr. 12, 76131 Karlsruhe, Germany \\ E-mail: thomas.hochrainereizbs.uni-karlsruhe.de
}

\section{Michael Zaiser}

The University of Edinburgh, Center for Materials Science and Engineering, King's Buildings, Sanderson Building, Edinburgh EH93JL, United Kingdom

E-mail: m.zaiser@ed.ac.uk

\begin{abstract}
In the context of recent proposals to use statistical mechanics methods for building a continuum theory of dislocation lines, mathematical modelling has to answer three essential questions: (i) What is the mathematical object representing the single dislocation as basic "particle"? (ii) What is the law of motion of this object? (iii) What is the mathematical nature of a dislocation density built of such objects? If a mathematically rigorous answer to these questions can be given, one may expect to derive the kinetic evolution equation for such a density solely from its definition and a conservation law. We present a method for deriving classical and non-classical dislocation density measures as well as their evolution equations from the properties of single dislocations, using the close connection between differential forms and geometrical objects such as dislocation lines. Several dislocation density measures are compared in view of their ability to represent vital aspects of the statics and dynamics of discrete dislocation configurations. A dislocation density measure which considers line directions and curvatures is defined as differential form, and it is shown that its evolution correctly represents the essential features of dislocation motion.
\end{abstract}

International Conference on Statistical Mechanics of Plasticity and Related Instabilities

31 August - 2 September 2005

Bangalore, India

\footnotetext{
* Speaker.

${ }^{\dagger}$ We gratefully acknowledge partial financial support from the European Commission through the Marie-Curie Research Training Network SizEDEPEN (contract no. MRTN-CT-2003-504634)
} 


\section{Introduction}

In the last years there has been a growing interest in physically based continuum theories of dislocations. This development has been driven by two problems, namely the explanation of size effects in small-scale structures and the long-standing question of the mechanisms which govern dislocation patterning. A continuum treatment of both problems seems only sensible if the continuum theories are built directly on the discrete unit of plastic deformation, i. e. the dislocation. An important step towards continuum modelling of interacting dislocation systems was done by Groma [2], who used the analogy between charged point particles in a plane and straight edge dislocations moving on a single slip system. By adopting averaging methods from statistical physics, Groma arrived at a density-based representation of the dynamics of such systems. After presenting a mean field theory in the original paper, the treatment was refined in several follow-ups to include short range interactions, see e. g. [3]. Comparison of discrete dislocation dynamics simulations and numerical solutions of the density-based dynamical equations demonstrates that the refined models provide a faithful representation of the discrete dynamics of two-dimensional dislocation systems, even if nontrivial boundary conditions are imposed [12].

It seems quite promising to use a similar approach to arrive at a continuum description of more realistic, three-dimensional dislocation systems. The three-dimensional generalization of such theories is, however, a challenging task in view of the line-like character of dislocations which renders the adoption of methods originally developed for interacting particles somewhat non-trivial. The most fundamental question in this regard is how to characterize the local dislocation state. The classical dislocation density tensor does not carry enough information for this purpose as was already noted by Köner [8]. In the pioneering work of El-Azab [1], the dislocation state was characterized by direction and velocity-dependent phase space distributions and density functions. However, as will be explained below, this may lead to a description in terms of unconnected dislocation segments which represent the evolution of a system of connected lines only in an incomplete manner. Nevertheless, the idea of characterizing dislocation configurations in terms of objects defined on higher-dimensional spaces seems promising. The question is how higher-dimensional extensions of the classical representation of a three-dimensional dislocation configuration (i.e., the representation in terms of the dislocation density tensor) may be constructed.

The application of statistical mechanics to two-dimensional systems of straight edge dislocations relies heavily on their equivalence to particle systems in two dimensions. For such systems it is well known (i) how to treat the point particles as a singular density written in terms of $\delta$ distributions, (ii) how to do averages over the single objects, as well as (iii) how to handle the averaged object as a density. In this paper we will mainly be concerned with the question how to generalize the pertinent concepts and methods to curved lines and their averages. This is most naturally done by using the language of differential forms and currents. As this leads to a formulation which is independent of the dimension of the underlying space, it also constitutes an ideal starting point for systematic generalizations as shown in Sections 5 and 6.

The idea of applying the language of differential forms to the continuum theory of dislocations is not new. Already Kröner was aware of the nature of the dislocation density tensor as a differential form [8]. The language of differential forms may be exploited for purely formal exercises, see e.g. the formal translation of the classical three dimensional theory to a four dimensional space-time in 
[11]. However, in the following we shall demonstrate that it can also be used as a powerful tool for overcoming structural deficiencies of the dislocation density tensor and obtaining an improved description of dislocation systems.

\section{Differential Forms}

Differential forms are a standard tool in differential topology and differential geometry. They are also widely used in different branches of physics, as for example in Maxwell theory. We will therefore just give a collection of facts about how to do calculus with differential forms, which turn vector analysis into a rather algebraic formalism working "automatically". A deeper understanding of the meaning of differential forms as representations of continuously distributed subspaces (submanifolds) will be addressed in the section on currents.

A very simplified heuristic definition might envisage differential forms of order $p$ as objects which may be integrated over $p$-dimensional spaces. From three-dimensional Maxwell theory we may take as examples $(i)$ the electric potential which is only meaningfully evaluated at points (zerodimensional spaces) and is thus a 0 -form, (ii) the electrical field which may be integrated over lines as a 1-form, (iii) the magnetic flux density which may be integrated over surfaces and therefore is considered as a 2-form and (iv) densities which define 3-forms and can be integrated over volumes. This is reflected in the spatial part of the physical dimensions of the objects, e. g. in the case above we have (i) the electric potential $[\mathrm{V}]$, (ii) the electrical field $\left[\mathrm{V} \cdot \mathrm{m}^{-1}\right]$, (iii) the magnetic flux density $\left[\mathrm{Wb} \cdot \mathrm{m}^{-2}\right]$ and (iv) the mass-density $\left[\mathrm{kg} \cdot \mathrm{m}^{-3}\right]$. Using this as a (very hand-waving) criterion for the mathematical representation of physical objects, we immediately see that a dislocation density with dimension $\left[1 \cdot \mathrm{m}^{-2}\right]$ should be defined as a 2 -form.

\subsection{Vector fields and differential 1-forms}

Although the notions of manifolds, charts, tangent and cotangent bundles are the starting point of the following calculations, we will not go into any detail but rather adopt parts of the language as given. An important concept, however, is the understanding of tangent vectors $\left.X\right|_{p}$ (vectors annexed to a spatial point $p$ ) as acting on functions $f$ as directional derivative at $p$ by $\left.X\right|_{p}(f)=$ $\left.X^{i} \frac{\partial}{\partial x^{i}}\right|_{p}(f)=X \cdot \nabla f(p)$. Here and in the following we adopt the Einstein summation convetion for automatically contracting over pairs of upper and lower indices. The underlying space is considered $n$-dimensional and for the above notion it makes no difference whether we think of it as a general $n$-dimensional manifold in a chart or simply of $\mathbb{R}^{n}$ with standard co-ordinates. We consider $\left.\frac{\partial}{\partial x^{i}}\right|_{p}$ as a local basis for tangent vectors and introduce the abbreviate notion $\left.\partial_{x^{i}}\right|_{p}$ for them.

In addition to tangent vectors we also can define co-vectors or 1-forms (sometimes also called Pfaffian forms) as locally linear mappings from a tangent space to the real numbers. Each 1-form $\omega$ is locally given by $n$ numbers $\omega_{i}(p)$ (similar to a vector) by setting $\omega_{i}(p)=\omega\left(\left.\partial_{x^{i}}\right|_{p}\right)$. Formally we can define a local basis $\left.d x^{i}\right|_{p}$ for co-tangent vectors by demanding $\left.d x^{i}\right|_{p}\left(\left.\partial_{x^{i}}\right|_{p}\right)=\delta_{j}^{i}$, with the Kronecker index $\delta_{j}^{i}$. Then it is obviously $\omega=\omega_{i} d x^{i}$. Best known example of 1-forms are those arising as differentials of a function $f$ denoted by $d f=\partial_{x^{i}} f d x^{i}$. They act on a vector $X$ in a dual way to the vector acting on the function $f$ by $d f(X)=X^{i} \partial_{x^{i}} f$. 


\section{Higher-order differential forms}

A (differential) $p$-form is a co-variant tensor $A=A_{i_{1} \cdots i_{p}} d x^{i_{1}} \otimes \cdots \otimes d x^{i_{p}}$ of order $p$ which obeys for each permutation $\pi$ of order $p$ the relation $A\left(X_{\pi(1)}, \ldots, X_{\pi(p)}\right)=\operatorname{sign} \pi A\left(X_{1} \ldots X_{p}\right)$. Functions are considered as 0 -forms. We define an alternating product $\wedge$ of a $p$-form $\omega$ and a $q$-form $\theta$ as the $(p+q)$-form given by

$$
\omega \wedge \theta\left(X_{1}, \ldots, X_{p+q}\right)=\frac{1}{p ! q !} \sum_{\pi} \operatorname{sign} \pi \omega \otimes \theta\left(X_{\pi(1)}, \ldots, X_{\pi(p+q)}\right) .
$$

This product is associatve, thus $(\omega \wedge \theta) \wedge \eta=\omega \wedge(\theta \wedge \eta)$, and it holds for $\omega$ and $\theta$ as in (2.1), $\omega \wedge \theta=(-1)^{p q} \theta \wedge \omega$. We moreover receive a basis for the space of differential $p$-forms from the alternating products $d x^{i_{1}} \wedge \cdots \wedge d x^{i_{p}}$ of the basis of 1 -forms $d x^{i}$ by requiring the indices to be sorted by $i_{1}<\cdots<i_{p}$. Each $p$-form $\omega$ can then be written as $\omega=\omega_{i_{1} \cdots i_{p}} d x^{i_{1}} \wedge \cdots \wedge d x^{i_{p}}$.

Besides the exterior multiplication of two forms, there is another important algebraic operation on differential forms, which is the inner multiplication by a vector-field $X$. For each $p$ form $\omega$ the inner multiplication with $X$ is the $(p-1)$-form $\mathrm{i}_{X} \omega$ defined by $\mathrm{i}_{X} \omega\left(X_{1}, \ldots, X_{p-1}\right)=$ $\omega\left(X, X_{1}, \ldots, X_{p-1}\right)$. In standard tensor notation this is a contraction of $X$ with the first index of $\omega$.

\section{Calculus on differential forms}

Two differential operators on differential forms are of special importance for the following: the exterior derivative $d$ and the Lie-derivative $\mathcal{L}$. The exterior derivative $d$ is defined by

$$
d \omega=d \omega_{i_{1} \cdots i_{p}} \wedge d x^{i_{1}} \wedge \cdots \wedge d x^{i_{p}}=\frac{\partial \omega_{i_{1} \cdots i_{p}}}{\partial x^{i}} d x^{i} \wedge d x^{i_{1}} \wedge \cdots \wedge d x^{i_{p}} .
$$

In standard three-space $d$ turns out to be the grad-, curl- and div- operator on 0-, 1- and 2-forms, respectively. As an easy consequence from standard calculus it is $d \circ d=0$.

As noted in the introduction to this section an important property of differential forms is that they can be integrated over appropriate sub-spaces. For spatial reasons we can not go in details about this but only state that on spaces with a standard volume form $d V$ each $n$-form can be written as $\omega=\rho d V$ with a function $\rho$ and can then be integrated like a usual function. In three-space the standard volume is given in standard co-ordinates as $d x^{1} \wedge d x^{2} \wedge d x^{3}$, which is more conveniently written as $d x^{1} d x^{2} d x^{3}$ when used in multiple integrals. The integral theorems of Gauß and Stokes turn out to be special cases of the general Stokes' theorem which says that for a differential $(n-1)$ form on a $n$-dimensional manifold $M$ with boundary $\partial M$ it is

$$
\int_{M} d \omega=\int_{\partial M} \omega
$$

Stokes theorem together with the fact that $d \equiv 0$ on $n$-forms easily yields the product rule

$$
\int_{M} d \omega \wedge \theta+(-1)^{p} \int_{M} \omega \wedge d \theta=\int_{\partial M} \omega \wedge \theta
$$

where $\omega$ is a $p$-form and $\theta$ a $(n-(p+1))$-form.

The Lie-derivative of a differential form in the direction of a vector-field $X$ can be defined by combining the exterior derivative and the inner multiplication as

$$
\mathcal{L}_{X} \omega=d \mathrm{i}_{X} \omega+\mathrm{i}_{X} d \omega .
$$


Similar to the product rule (2.4) we find for the Lie-derivative

$$
\int_{M} \mathcal{L}_{X} \omega \wedge \theta+\int_{M} \omega \wedge \mathcal{L}_{X} \theta=\int_{\partial M} \mathrm{i}_{X}(\omega \wedge \theta)
$$

If a standard volume density $d V$ is defined on the underlying space (thus each $n$-form $\omega$ can be written as $\omega=\rho d V$ ), it is possible to define the divergence of a vector-field $X$ by

$$
\mathcal{L}_{X} d V=d\left(\mathrm{i}_{X} d V\right)=\operatorname{div} X d V .
$$

With this we do have all necessary concepts at hand to reformulate the (purely kinematic part of the) classical continuum theory of dislocations in the language of differential forms.

\section{Kröner revisited}

We start with noting that already Kröner was well aware of the fact that the dislocation density tensor $\alpha$ conceptually emerges as a vector-valued (given by the Burgers vector) 2 -form, i. e. $\alpha=\alpha_{i j}{ }^{k} d x^{i} \otimes d x^{j} \otimes \partial_{x^{k}}$ with $\alpha_{i j}{ }^{k}=-\alpha_{j i}{ }^{k}$. But as commonly done in three dimensions he uses the totally anti-symmetric symbol $\varepsilon^{i j k}$ to make it a more convenient two-tensor $\alpha=\alpha^{i j} \partial_{x^{i}} \otimes \partial_{x^{i}}$ by setting $\alpha^{i j}=\varepsilon^{i k l} \alpha_{k l}^{j}$. We will not go into more details about this transformation here, but only state that it makes implicitely use of the natural co-ordinates and the standard metric in $\mathbb{R}^{3}$. A purely kinematic theory, however, should be independent of the chosen metric, as is the formulation with differential forms.

In order to simplify the calcualtions we assume that $\alpha$ is only representing dislocations of one glide system, thus the Burgers vector $b=b^{k} \partial_{x^{k}}$ is fixed and $\alpha$ can be split into the tensor product of $b$ and a 2-form $\bar{\alpha}$ as $\alpha=\bar{\alpha} \otimes b=\bar{\alpha}_{i j} b^{k} d x^{i} \wedge d x^{j} \otimes \partial_{x^{k}}$. Because the Burgers vector $b$ is taken to be constant all differential operations need only be applied to the 2-form $\bar{\alpha}$. As long as only single glide is allowed this also naturally splits the plastic distortion tensor $\beta^{\mathrm{pl}}$ into $\beta^{\mathrm{pl}}=\bar{\beta}^{\mathrm{pl}} \otimes b=$ $\bar{\beta}_{i}^{\mathrm{pl}} b^{j} d x^{i} \otimes \partial_{x^{k}}$. We are now able to translate the classical equations for the dislocation density tensor, as found e. g. in [8] and [9], into the language of differential forms.

\begin{tabular}{|l|l|}
\hline$\alpha=\operatorname{curl} \beta^{\mathrm{pl}}$ & $\alpha=d \beta^{\mathrm{pl}}$ \\
\hline $\operatorname{div} \alpha=0$ & $d \alpha=0$ \\
\hline$\partial_{t} \alpha=-\operatorname{curl}(v \times \alpha)$ & $\partial_{t} \alpha=-d\left(\mathrm{i}_{\nu} \alpha\right)=-\mathcal{L}_{\nu} \alpha$ \\
\hline
\end{tabular}

Table 1: Important equations of the field theory of dislocations in classical formulation (left) and in terms of differential forms (right)

In Table 1 the two formulations are compared, where in both cases all differential operators are acting solely on the tensorial part represented by the according first indices. Listed from top to bottom the three equations compared in Table 1 can be viewed as the definition of $\alpha$, the representation of the fact that dislocations may not end inside a crystal and the conservation law for the net Burgers vector during plastic deformation.

Already Kröner noted in [8] that the first equation "is the continuum version of the definition [...] in which dislocations are defined as edge lines of interior surfaces over which slip has occurred" and that "clearly a surface and its edge line are connected by the curl-operation (Stokes' 
theorem)". Indeed to understand this statement in full and to be able to draw similar conclusions for the other two equations we need the generalizition of $\delta$-distributions, named currents.

\section{Distributions and Currents}

We begin this section by taking a closer view on the nature of $\delta$-distributions. It is usual to think of them as a sort of generalized function (in a literal sence) that can be approximated by smooth functions which concentrate around a point and the integral over each of them is one. More precisely, however, we should rather view them as functionals acting on vector spaces of smooth functions. Both viewpoints have their merits and their draw-backs and we will formally adopt the latter one but use the first one to understand the calculus defined on generalized functions.

We consider the delta distribution $\delta\left(r-r_{0}\right)$ at a point $r_{0}$ to be acting on functions $f$ by $\delta\left(r-r_{0}\right)(f)=f\left(r_{0}\right)$. Thus it assigns to each function its value at the point $r_{0}$. More conveniently this action is written as an integral like

$$
\delta\left(r-r_{0}\right)(f)=\int \delta\left(r-r_{0}\right) \cdot f(r) d V .
$$

In the language of differential forms we would therefore rather understand $\delta$ as a generalized $n$-form, $\delta\left(r-r_{0}\right) d V$, than as a generalized function. This corresponds to the canonical way of representing an average over point particles by a density, i. e. a volume form and not as a "potential".

It is a well known that the kinematics of point paritcles, to which an average velocity $v$ can be assigned, yields a conservation law for the evolution of the average density $\rho$ of them as $\partial_{t} \rho=$ $-\operatorname{div}(\rho v)$, when no sources of paritcles are present. We will see that this equation also makes sense for the single particle represented as $\delta$-distribution. To see this we turn the average density $\rho$ into a functional acting on smooth functions by integrating against, thus $\rho(f)=\int \rho f d V$. Then we find

$$
\begin{aligned}
\left.\partial_{t}\right|_{t=0} \rho(f) & =\int \partial_{t} \rho f d V \\
& =-\int \operatorname{div}(\rho v) f \\
& =-\int \operatorname{div}(f \rho v)-\rho \cdot v(f) d V \\
& =\int \rho \cdot v(f) d V \\
& =\rho(v(f))
\end{aligned}
$$

The first integral in the third line vanishes due to Gauß' integral theorem if $f$ is taken to vanish near the boundary of the area integrated over, which is an integral feature of the function spaces generalized functions are supposed to act upon.

On the other hand, if the single particle is moving, thus $r_{0}=r_{0}(t)$ with $\partial_{t} r_{0}(t)=v$, we can differentiate Equation (4.1) and do easily find

$$
\begin{aligned}
\left.\partial_{t}\right|_{t=0} \delta\left(r-r_{0}(t)\right)(f) & =\lim _{t \rightarrow 0} \frac{1}{t} f\left(r_{0}(t)\right)-f\left(r_{0}(0)\right) \\
& =v(f)\left(r_{0}(t)\right) \\
& =\delta\left(r-r_{0}(0)\right)(v(f)) .
\end{aligned}
$$


Thus the averaged density viewed as a functional fullfills the same differential equation like the single particle. We furthermore notice that we can rewrite the preceeding as

$$
\begin{aligned}
\partial_{t}(\rho d V) & =-\operatorname{div}(\rho v) d V=-\mathcal{L}_{v}(\rho d V) \text { and } \\
\partial_{t} \rho(f) & =\rho\left(\mathrm{i}_{\mathrm{v}} \mathrm{df}\right)=\rho\left(\mathcal{L}_{v} f\right) .
\end{aligned}
$$

We now turn to a generalizing of $\delta$-distributions by considering functionals acting on differential forms. Like noted in the introduction to differential forms, one of their main properties is that they can be integrated over sub-manifolds of the according dimension. This does in turn directly yield that each sub-manifold $N$ of dimension $q$ does define a functional on differential $q$-forms $\theta$ in virtue of

$$
N(\theta)=\int_{N} \theta
$$

Similarly does every $p=(n-q)$-form $\omega$ define a functional on $q$-forms via

$$
\omega(\theta)=\int_{M} \omega \wedge \theta
$$

Functionals on (admissible) spaces of differential forms are called currents and we see that this concept includes sub-manifolds and differential forms. Currents on the space of $q$-forms are for obvious reasons said to have dimension $q$ and degree $(n-q)$. We have already seen an example of this in the relation between points (i. e. 0 -dimensnional sub-manifolds) and $n$-forms when discussing the concept of $\delta$-distributions. In view of the dislocation case we can already anticipate the correspondance between surfaces over which slip has occurred and the (vector-valued) 1-form $\beta^{\mathrm{pl}}$ as well as between dislocation lines and the (vector-valued) 2-form $\alpha$. That the curl-operation does correspond to the understanding of dislocations as boundary of the slipped surfaces and that Stokes' theorem indeed defines this connection can be seen as follows.

Stokes' theorem (2.3) directly allows to define the boundary of a current $\gamma$ of dimension $q$ in analogy to the sub-manifold case by its action on a $(q-1)$-form $\theta$, according to

$$
\partial \gamma(\theta)=\gamma(d \theta)
$$

On the other hand we can define the exterior derivative $d$ of a current in analogy to the one on differential forms from the product rule (2.4). The differential forms currents are acting upon are defined such that the boundary integral in (2.4) vanishes, wherefore the exterior derivative of a current $\gamma$ of degree $p$ is defined as

$$
d \gamma(\theta)=-(-1)^{p} \gamma(d \theta)=(-1)^{(p+1)} \partial \gamma(\theta) .
$$

Hence the generalized exterior derivative equals the generalized boundary operator up to a sign. For currents of odd degree both are the same and we find that the definition of the dislocation density tensor as the curl of the plastic distortion, $\alpha=d \beta^{\mathrm{pl}}$, indeed directly corresponds the representation of the the slipped area and its boundary as (vector valued) currents in $\alpha=\partial \beta^{\mathrm{pl}}$. Similarly the postulation of $\alpha$ being free of divergence does indeed turn to the condition that dislocations do not end inside the crystal, saying that they do not have a boundary as a submanifold and therefore $d \alpha=-\partial \alpha=0$, when turned to a current. 
We give a sketch of the proof for the fact that the evolution equation $\partial_{t} \alpha=-\operatorname{curl}(v \times \alpha)=$ $-\mathcal{L}_{v} \alpha$ is applicable to the single dislocation viewed as current. This is a special case of a result found in [4] for sub-manifolds of arbitrary dimension. Let therefor $c(t, u)=c_{t}(u)$ be a parametrization of a moving curve-segment in $M$, which is at each time $t$ parametrized by a parameter $u$ running from 0 to $U$. We denote with $S_{t}$ the surface swept by $c$ since time $t=0$ and with $c_{t}^{0}$ and $c_{t}^{U}$ the curves characterized by the paths of the according end-points of the curve. Let furthermore $v(u)=\left.\partial_{t}\right|_{t=0} c(t, u)$ be the velocity describing the motion of $c$ at time $t=0$. With this we find for the time-derivative of the induced time-dependent current

$$
\begin{aligned}
\left.\partial_{t}\right|_{t=0} c_{t}(\omega) & =\lim _{t \rightarrow 0} \frac{1}{t}\left(\int_{c_{t}} \omega-\int_{c_{0}} \omega\right) \\
\stackrel{\text { Stokes }}{=} & \lim _{t \rightarrow 0} \frac{1}{t}\left(\int_{S_{t}} d \omega-\left(\int_{c_{t}^{0}} \omega-\int_{c_{t}^{U}} \omega\right)\right) \\
& =\int_{c_{0}} d \omega(v, \cdot)+\left.\omega(v)\right|_{(0,0)} ^{(0, U)} \\
\stackrel{\text { Stokes }}{=} & \int_{c_{0}} \mathrm{i}_{v} d \omega+\int_{c_{0}} d \mathrm{i}_{v} \omega \\
& =c_{0}\left(\mathcal{L}_{v} \omega\right) .
\end{aligned}
$$

Considering the product rule for the Lie-derivative (2.6), the Lie-derivative for a current $\gamma$ is defined as

$$
\mathcal{L}_{X} \gamma(\omega)=-\gamma\left(\mathcal{L}_{X} \omega\right)
$$

and consequently we just found that the literal translation of the motion of a curve $c$ into the evolution of a current $\alpha$ yields $\partial_{t} \alpha=-\mathcal{L}_{\nu} \alpha$.

Summing up we have seen that the three equations listed in Table 1 do apply for the singular object as well as for the averaged counterparts. However, this also implies that a dislocation system and its evolution is only correctly described by these equations if either the dislocation actually form smooth line bundles (nearby dislocations are nearly parallel and have the same Burgers vector) or the spatial resolution is fine enough to display each dislocation separately. The first case is rather untypical as dislocations tend to form irregular networks while the latter case is equivalent to a discrete dislocation representation. The statistical nature of the local orientation distribution in dislocation networks therefore asks for more refined dislocation density measures, allowing for a truely averaged description. Such a dislocation density measure, however, should surely keep some structural features of the classical theory. In the following we will give a brief overview of yet proposed refined measures and discuss their relation to the classical theory. Furthermore we propose two ways of generalizing the classical theory in the language of differential forms which are called statistical and deterministic for reason which will be explained below. The refined measures found in the literature are considered as statistical ones.

\section{5. "Statistical" generalizations}

The first statistical dislocation density measure was proposed by Kosevich [7]. He classified dislocations at each point by their line-direction and defines a density function $\rho(p, l)$ depending on the spatial point $p$ and the direction $s$ as giving the number of dislocations at $p$ whose orientation 
is contained in a solid angle element $d S$ around $s$. Thus the dislocation density measure reads $\alpha=\rho(p, s) d S$. A very similar measure which originally also accounts for a velocity distribution at each point was later proposed by El-Azab [1]. After averaging the phase space distribution $\phi(p, s, v) d V d S d Q$ over the velocity space - or collapsing the velocity distribution by assuming overdamped dislocation motion - this reads $\alpha=\rho(p, s) d V d S$ with $\rho=\int_{v} \phi(p, s, v) d Q(v)$. The density $\rho$ here carries the same information like in the case of Kosevich, but does count dislocations of a certain direction $s$ within a spatial volume element $d V$ around a point $p$. Actually the difference between both is best understood in the language of differential forms which additional brings out an important draw-back of both definitions. Both measures are defined on the configurational space made up of the crystal points and all possible directions at each point. They can therefore be seen as consisting of a spatial and a directional part. In the definitions this is displayed in the volume element $d V$ and the solid angle element $d S$. In terms of differential forms, however, this means that the measure of Kosevich is spatially a 0-form (like e. g. a potential) and El-Azabs measure is spatially a 3-form (like a density of point particles). This is also reflected in the evolution law derived by El-Azab who treats the appearing density functions $\phi$ and $\rho$ like those for a conservative system of angular dependend point particles. This results in a Liouville equation for $\phi$ and the classical conservation law $\partial_{t} \rho=-\operatorname{div}(\rho v)$ for the segment density. To the authors knowledge there is no evolution law given by Kosevich.

It is rather straight forward to give a similar description which accounts for the line like character of the dislocations by assigning to each point in the phase space $(p, s)$ a spatial unit vector $l(s)$ with the according direction. Then $\alpha=\rho(p, s) \mathrm{i}_{l} d V d S$ defines a spatial 2-form. We need to extend the spatial velocity $v$ to a generalized velocity on the configuration space $V=\left(v,\left(\nabla_{l} v\right)^{\perp}\right)=(v, \vartheta)$, where the second component $\vartheta$ accounts for the rotation of the dislocation segments during the motion of the dislocation. The evolution equation for this measure reads

$$
\partial_{t} \alpha=-P\left(\mathcal{L}_{V} \alpha\right),
$$

where $P$ denotes a projection operator ensuring that it is $\partial_{t} \alpha=\partial_{t} \rho(p, s) \mathrm{i}_{l} d V d S$. In other words any evolving curvature is ignored in the same fashion as it is in the definition of the measure. Actually this yields the same evolution equation as given by El-Azab. It seems therefore not too surprising that it was shown in [6] that this evolution law can only describe the evolution of a dislocation system if the dislocations are and stay straight, which is only possible if the absolut value of the velocity is constant on the whole configuration space. This is obviously not acceptable when aiming at a continuum theory of dislocations.

Before going for a solution of this problem we will state why we called the preceeding dislocation density measures statistical. If a volume density is defined on the underlying space (like implicitely in the usual calculus) there is a canonical way to turn a 2-form into a vector field by virtually inverting the inner multiplication used above to create a 2 -form from a vector field. If this is done for the classical dislocation density this vector field does in principal allow for recovering the dislocation lines as the flux lines (integral curves) of this vector-field. This is not possible with the measures introduced above, because no information on the curvature is available which would be needed to reconstruct the dislocation lines. This remaining "uncertainty" about the true dislocation state gives rise to the term statistical. The advantage of a statistical measure clearly is that it does not impose restrictions on "representable" dislocation distributions, while the restricted 
applicability of the classical theory indeed corresponds to the rare cases where the integral curves actually recover the dislocations.

However, that the curvature is not known is also the main reason why these descriptions do only work for trivial velocity distributions. As was already pointed out in [13] this can easily be seen in that the density function $\rho(p, l)$ can not distinguish between a distribution of straight dislocations with random direction or randomly distributet dislocation loops of a fixed radius. However, for a spatially constant absolut value of the velocity, the density $\rho$ would in the first case stay constant while it would have to increase in the second one. It was therefore proposed in [5] that the curvature should be included in the configuration space. Then the configuration space is made up of points $p$, directions $s$ and curvatures $k$ and we can extend the density defined at last to

$$
\alpha(p, s, k)=\rho(p, s, k) \mathrm{i}_{l} d V \mathrm{i}_{k} d S d K .
$$

This measure additionally claasifies dislocations by their curvature but still is a statistical measure as now the higher order curvature would be needed to reconstruct the dislocation lines. For this case the generalized velocity $V$ has additionally to include a term accounting for the curvature change during the motion of the dislocation, which is (necessarily) given by $\mathfrak{k}=\langle v, k\rangle k+\nabla_{l} \vartheta$, and yields $V=(v, \vartheta, \mathfrak{k})$. The brackets $\langle\cdot, \cdot\rangle$ denote the scalar product of two vectors. The evolution equation is again given by

$$
\partial_{t} \alpha=-P\left(\mathcal{L}_{V} \alpha\right) .
$$

where the projection operator $P$ has to be adjusted accordingly. This evolution equation can be turned to an evolution equation for $\rho(p, s, k)$, which is in [6] shown to be given by

$$
\partial_{t} \rho=-\operatorname{Div}(\rho V)+\langle v, k\rangle \rho .
$$

Here Div denotes the divergence operator on the configuration space. This evolution equation can of course distinguish between distributions made up solely of straight dislocations or including curved dislocations and the term $\langle v, k\rangle \rho$ will account for changes of total line length in the system due to the motion of curved lines. However, to capture the evolution of a dislocation system correctly we do again have to impose conditions on the nature of the velocity fields, saying the absolute value of the velocity may not depend on the line direction nor on the curvature but solely on the spatial point. This still is a serious restriction for a general theory of dislocations as dislocations may have different mobilities depending on their character (edge, screw or mixed). Furthermore it seems natural to include line tension effects into the velocity, what would make its absolut value depending on the local curvature. One might think that a straight forward extension of this approach in that the configuration space would include higher order curvatures and allowing for a variation of the curvatures of the highest order in the definition of $\alpha$ (rendering the approach a statistical one in the sense explained above) could further attenuate the restrictions imposed on the velocity; but it was shown in [6] that a statistical approach of arbritary order would have to make the same restriction to $v$ as the one including just first order curvatures. Although the mistake made would presumably become smaller with approaches of higher order this does not seem to be a promising way to arrive at a managable description, as the dimension of the configuration space would further increase. We therefore turn to an extension of the classical dislocation density measure which is deterministic in the sense that it allows to reconstruct the dislocation lines. 


\section{Deterministic generalization}

We start this section with a short review of a reformulation of the classical dislocation density evolution given by Sedláček und Kratochvíl [10]. As was noted before, the dislocation density tensor $\alpha$ as a vector valued 2-form allows to determine a vector field $R$ for which $\alpha=b \otimes \mathrm{i}_{R} d V$. If there is a Riemannian metric, i. e. a local scalar product given, this vector-field can be written as $R=\|R\| \cdot \frac{R}{\|R\|}$ and with $\rho=\|R\|$ and $l=\frac{R}{\|R\|}$ we may write $\alpha=b \otimes \rho \mathrm{i}_{l} d V$. The evolution of $\alpha$ can then be described by the evolution of the density $\rho$ and of the direction $l$. Sedlacek and Kratochvil derived according formulas for the two-dimensional situation of dislocations moving only within their glide plane. Parametrizing the directional space by an angle $\varphi$ they find

$$
\begin{aligned}
& \partial_{t} \rho=-\operatorname{div}(\rho v)+\rho\|v\| k \\
& \partial_{t} \varphi=\nabla_{l}\|v\|-\|v\| \operatorname{div} l,
\end{aligned}
$$

with $k$ being the curvature of the flux lines of $R$. Comparing this to the evolution equation 5.4 derived for the curvature-including statistical theory, we find that the evolution of $\rho$ looks nearly the same, but that there is no complement to the directional evolution in the statistical theories. Indeed the according evolution is deliberately ignored by applying the projection operator $P$ in order to conserve the nature of a statistical measure. It seems therefore appropriate to ask whether the unrequestedly evolving part should not be constituent of the definition of the dislocation density measure. For the measure defined on the configuration space made up of points and directions, originally defined as $\alpha=\rho(p, s) \mathrm{i}_{l}(s) d V d S$, this would yield

$$
\alpha=\rho(p, s) \mathrm{i}_{l}(s) d V \mathrm{i}_{k}(p, s) d S,
$$

with a curvature vector $k$ depending on space and direction. The precondition that dislocations do not end inside the crystal could then be directly transferred as $d \alpha=0$, or equivalently $\operatorname{Div}(\rho \cdot(l, k))=$ $\operatorname{Div}(\rho \cdot L)=0$. With the generalized velocity $V=(v, \vartheta)$ the evolution equation is therefore given like in the classical case by

$$
\partial_{t} \alpha=-\mathcal{L}_{V} \alpha .
$$

The sketch of a two-dimensional example shall elucidate the evolution determined from this equation. We consider only dislocations of the same Burgers vector moving within their glide plane. The glide plane is taken to be the $x-y$-plane and the space of directions at each point is characterized by the angle $\varphi$ the dislocation segments form with the $x$-axis. The velocity vector $v$ and the curvature vector $k$ are then both characterized by a (pseudo-) scalar as their direction is fixed to be orthogonal to the line. This yields

$$
\begin{aligned}
L(x, y, \varphi) & =\cos \varphi \partial_{x}+\sin \varphi \partial_{y}+k \partial_{\varphi} \\
V(x, y, \varphi) & =v \sin \varphi \partial_{x}-v \cos \varphi \partial_{y}-L(v) \partial_{\varphi}
\end{aligned}
$$

and we find

$$
\begin{aligned}
\partial_{t} \rho & =-\operatorname{Div}(\rho \cdot V)+v k \rho \\
\partial_{t} k & =-v k^{2}+L(L(v))-V(k) .
\end{aligned}
$$

A derivation of these formulas as well as a detailed discussion of the underlying conservation law will be given in a following paper. 


\section{Summary and Perspectives}

The concept of currents was used to show how evolution equations for differential forms can be directly cast from the equations of motion of a single geometric object. We used a differential form formalism to extend the classical dislocation density measure to higher dimensional descriptions which are able to reflect more details of a dislocation distribution. We distinguish two kinds of dislocation density measures, deterministical and statistical ones. The deterministical measures are not able to represent arbitrary discloation states but yield exact evolution equations for admissible distributions, while the statistical measures may correctly represent a general dislocation state, but impose restrictions on admissible velocity distributions in order to capture the evolution correctly. Future work will have to address the question to which extend these restrictions may be acceptable for a statistical mechanics description of plasticity.

\section{References}

[1] A. El-Azab. Statistical mechanics treatment of the evolution of dislocation distributions in single crystals. Phys. Rev. B, 61:11956-11966, 2000.

[2] I. Groma. Link between the microscopic and mesoscopic length-scale description of the collective behavior of dislocations. Phys. Rev. B, 56:5807-5813, 1997.

[3] I. Groma, F. F. Csikor, and M. Zaiser. Spatial correlations and higher-order gradient terms in a continuum description of dislocation dynamics. Acta Mater., 51:1271-1281, 2003.

[4] A. N. Hirani. Discrete Exterior Calculus. Thesis, California Institute of Technology, Pasadena, 2003.

[5] T. Hochrainer. A continuum theory of dislocation motion. In N. M. Ghoniem, editor, Proc. of $2^{\text {nd }}$ Int. Conf. on Multiscale Materials Modeling, pages 210-212, Los Angeles, US, 2004. Univ. of California Los Angeles.

[6] T. Hochrainer. unpublished. 2005.

[7] A. M. Kosevich. Dislocations in Solids, volume 1, pages 33-142. North Holland, Amsterdam, 1979.

[8] E. Kröner. Continuum theory of defects. North Holland Publ. Comp., 1981.

[9] T. Mura. Continuous distribution of moving dislocations. Phil. Mag., 8:843-857, 1963.

[10] R. Sedláček, J. Kratochvíl, and E. Werner. The importance of being curved. Phil. Mag., 83:3735-3752, 2003.

[11] K. Yamasaki and H. Nagahama. Hodge duality and continuum theory of defects. J. Phys A: Math. Gen., 32:L475-L481, 1999.

[12] S. Yefimov, I. Groma, and E. van der Giessen. A comparison of a statistical-mechanics based plasticity model with discrete dislocation plasticity calculations. J. Mech. and Phys. Solids, 52:279-300, 2004.

[13] M. Zaiser and T. Hochrainer. Some steps towards a continuum representation of 3d dislocation systems. Scripta Mater., 54:717-721, 2006. 